\title{
Radiographic Mastoid and Middle Ear Effusions in Intensive Care Unit Subjects
}

\author{
Phillip Huyett MD, Yael Raz MD, Barry E Hirsch MD, and Andrew A McCall MD
}

\begin{abstract}
BACKGROUND: This study was conducted to determine the incidence of and risk factors associated with the development of radiographic mastoid and middle ear effusions (ME/MEE) in ICU patients. METHODS: Head computed tomography or magnetic resonance images of 300 subjects admitted to the University of Pittsburgh Medical Center neurologic ICU from April 2013 through April 2014 were retrospectively reviewed. Images were reviewed for absent, partial, or complete opacification of the mastoid air cells and middle ear space. Exclusion criteria were temporal bone or facial fractures, transmastoid surgery, prior sinus or skull base surgery, history of sinonasal malignancy, ICU admission < 3 days or inadequate imaging. RESULTS: At the time of admission, $3.7 \%$ of subjects had radiographic evidence of ME/MEE; $10.3 \%(n=31)$ of subjects subsequently developed new or worsening ME/MEE during their ICU stay. ME/MEE was a late finding and was found to be most prevalent in subjects with a prolonged stay $(P<.001)$. Variables associated with ME/MEE included younger age, the use of antibiotics, and development of radiographic sinus opacification. The proportion of subjects with ME/MEE was significantly higher in the presence of an endotracheal tube $(22.7 \%$ vs $0.6 \%, P<.001)$ or a nasogastric tube $(21.4 \%$ vs $0.6 \%, P<.001)$. CONCLUSIONS: Radiographic ME/MEE was identified in $10.3 \%$ of ICU subjects and should be considered especially in patients with prolonged stay, presence of an endotracheal tube or nasogastric tube, and concomitant sinusitis. ME/MEE is a potential source of fever and sensory impairment that may contribute to delirium and perceived depressed consciousness in ICU patients. Key words: nosocomial infections; mastoid effusion; middle ear space effusion; intensive care unit; endotracheal intubation; nasogastric intubation; Lund-Mackay score. [Respir Care 2017;62(3):350-356.

(C) 2017 Daedalus Enterprises]
\end{abstract}

\section{Introduction}

Otitis media with effusion or a middle ear effusion (MEE) most often represents the accumulation of transudate in response to negative pressure and/or inflammation within

Drs Huyett, Hirsch, and McCall are affiliated with the Department of Otolaryngology-Head and Neck Surgery, University of Pittsburgh Medical Center, Pittsburgh, Pennsylvania; Dr Raz is affiliated with the Department of Otolaryngology-Head and Neck Surgery, Veteran's Affairs Hospital Pittsburgh, Pittsburgh, Pennsylvania.

Dr Huyett presented a version of this paper at the Triological Society at the Combined Otolaryngology Sections Meeting held May 21, 2016, in Chicago, Illinois.

The authors have disclosed no conflicts of interest.

The statistical analysis performed in this project was supported by the National Institutes of Health through Grant Number UL1TR000005. the middle ear space. ${ }^{1}$ Mastoid effusions (ME) occur in conjunction with MEE, because the 2 spaces are continuous with one another. Unlike MEE, ME are not detected on clinical exam but are readily noted on imaging. The most common sequelae of ME/MEE are related conductive hearing loss and the progression to acute otitis media.

Because of the orientation, length, and function of the Eustachian tube, MEE and acute otitis media are exceptionally common findings in children but are rare in adults and should prompt concern regarding disorders of the nose, nasopharynx, or ear itself. In the general pediatric popu-

Correspondence: Phillip Huyett, MD, University of Pittsburgh Medical
Center, Department of Otolaryngology-Head \& Neck Surgery, 200 Lo-
throp St, Suite 500, Pittsburgh, PA 15213. E-mail: huyettpa@ upmc.edu

DOI: $10.4187 /$ respcare. 05172 
lation, $12-27.1 \%$ of patients are incidentally noted to have ME or MEE on magnetic resonance imaging (MRI) performed for nonotologic reasons. ${ }^{2-4}$ In contrast, one study of 100 adults undergoing MRI for neurologic indications found that only $1 \%$ of subjects had otitis media with effusion. ${ }^{5}$

Previous studies have suggested that the ICU population represents a subgroup of adults who are at increased risk of MEE. ${ }^{6-10}$ Proposed mechanisms have included supine positioning, depressed level of consciousness and presence of foreign bodies such as nasogastric tubes. Given the frequency with which the ICU population undergoes imaging, review of available head computed tomography (CT) and MRI offers the opportunity to incidentally diagnose acute otitis media or ME/MEE. ${ }^{11}$ To our knowledge, no study has examined the radiographic incidence of ME/MEE in the ICU population.

Although other nosocomial infections, such as ventilator-associated pneumonia and urinary tract and central lineassociated bloodstream infections, appropriately draw more attention in the critical care literature, acute otitis media is a potential, albeit uncommon, cause of fever in the ICU patient. ${ }^{12,13}$ This patient population is probably more susceptible to complications related to acute otitis media given that the infection is prone to go unrecognized.

Benign MEE, on the other hand, can cause hearing loss that may contribute to delirium or perceived lesser consciousness by the medical care team. ${ }^{14}$ Addressing this modifiable cause of sensory impairment can greatly benefit the patient. Thus, it is important for the otolaryngologist and critical care physician to have an appreciation for the radiographic findings of the ear in the ICU patient. The objective of this study, therefore, was to determine the incidence of and risk factors associated with the development of ME/MEE among a large cohort of ICU subjects.

\section{Methods}

The clinical and radiographic data on 300 subjects admitted to the neurologic ICU at University of Pittsburgh Medical Center Presbyterian Hospital between April 18, 2013 and April 18, 2014 were retrospectively reviewed. The University of Pittsburgh Institutional Review Board approved the study. Subjects who underwent a head CT scan or MRI at the time of admission and at least one other point within the first $14 \mathrm{~d}$ of their ICU stay were included. Patients with temporal bone or facial fractures, transmastoid surgery, prior sinus or skull base surgery, sinonasal malignancy, an ICU stay of $<2$ nights $(3 \mathrm{~d})$, or an inpatient admission within 3 weeks leading up to the ICU stay were excluded.

Absent, partial (some aeration of the mastoid and middle ear spaces), or complete (no aeration) ME/MEE on imaging was determined on head CT or MRI. The para-

\section{QUICK LOOK}

\section{Current knowledge}

Although mastoid and middle ear effusions are uncommon in the general adult population, the ICU population may represent a unique patient cohort at increased risk of developing mastoid and middle ear effusions. Ear effusions are important to recognize, because of the associated conductive hearing loss that can lead to a perceived lesser level of consciousness and delirium in the recovering ICU patient. Less commonly, mastoid and middle ear effusions can progress to acute otitis media with associated infectious signs and symptoms.

\section{What this paper contributes to our knowledge}

Mastoid and middle ear effusions were identified in approximately $10 \%$ of ICU subjects undergoing head imaging for other reasons. There were strong correlations between the development of effusions and lengths of stay $>7 \mathrm{~d}$, concomitant sinus disease, and subjects who had been orotracheally or nasogastrically intubated. Given the frequency with which ICU patients undergo imaging, incidental notation of ear effusions can lead to appropriate workup and treatment.

nasal sinuses were scored using a validated staging system for sinonasal disease, the Lund-Mackay scoring system, using axial images only, as described previously. ${ }^{15,16} \mathrm{~A}$ combination of head CT and T2 weighted MRI was used when available. The presence of endotracheal (ETT) and nasogastric tubes on imaging was noted. Computed tomography and magnetic resonance images were scored from ICU day 1 to 14 . The day- 1 scan was considered the baseline from which subsequent scans were compared. For days $8-10$ and $11-14$, the last scan was chosen to represent these time frames given the decreased number of scans beyond 7 days. Each subject could therefore have a maximum of 9 scored scans on any combination of days.

SPSS 23 (IBM Corp, Armonk, New York) was used to perform the statistical analysis with a $P<.05$ considered significant. The incidence of worsening ME/MEE was defined as the development of worse radiographic opacification of the mastoid air cells or middle ear space (absent to partial, absent to complete, or partial to complete) at any point during the ICU stay compared with that on day 1. In this way, worsening ME/MEE represents a change from a subject's baseline and excludes those who were admitted with ME/MEE that did not progress. Similarly, worsening sinus disease was defined as a Lund-Mackay score that was higher at any point during the admission compared with the Lund-Mackay score on day 1. Comparison of incidence between the groups of categorical and continu- 
ous variables was performed with 2-sided Pearson's chisquare test and independent 2-sample $t$ test, respectively. Linear-by-linear association was used to test for trends of increasing or decreasing incidence by stay, age, and month of admission. Multivariable logistic regression was performed to determine the independent contributions of ETT, nasogastric tubes, and stay $>7 \mathrm{~d}$ to the development of ME/MEE.

\section{Results}

The 300 subjects were $53 \%$ male (159 of 300) and on average $63.5 \mathrm{y}$ old (range 16-91 y). The mean length of stay was $8.3 \mathrm{~d}$ (range 3-62 d), and subjects had an average of 5 sets of imaging (range 2-17). Stroke ( $n=96,32.0 \%)$, intracranial hemorrhage $(n=60,20.0 \%)$, subarachnoid hemorrhage $(n=58,19.3 \%)$, and subdural hematoma $(n=36,12.0 \%)$ were the most common admission diagnoses; $45.7 \%(n=137)$ of subjects underwent an intracranial surgery or angiographic intervention, and $32.0 \%$ $(n=96)$ were treated with nonprophylactic antibiotics (antibiotics not given perioperatively), and $22.0 \%$ of subjects had a history of immune system dysfunction or immunosuppression, which was most commonly caused by diabetes mellitus (92.4\%).

At the time of admission (day 1), 3.7\% (11 of 300) of subjects had an ME/MEE. Two of these subjects (18.2\%) developed worsening (complete) effusions, and the remaining 9 subjects $(81.8 \%)$ had resolution or nonprogression of their effusion. During the ICU stay, $10.3 \%$ of subjects (31 of 300) demonstrated worsening radiographic ME/MEE compared with the baseline/admission imaging study (Figs. 1 and 2); 29 (93.5\%) subjects developed ME/MEE from normal imaging on day 1 , and the other 2 subjects had progression of baseline ME/MEE.

There were 20 subjects with bilateral involvement, 7 subjects with only right ear, and 4 subjects with only left ear effusions. Complete opacification of the mastoid and middle ear space developed in 5.9\% (3/51) of the ears, whereas the remaining $94.1 \%$ developed partial opacification only.

The percentage of subjects with worsening opacification on a given day progressively increased to a peak of $30 \%$ on days 11-14 (Fig. 3). Similarly, the proportion of subjects who developed worsening effusions also had a positive correlation to length of stay $(P<.001$, Fig. 4). Subject age was found to have a statistically significant correlation with ME/MEE, wherein younger subjects had higher percentages of developing worse $\operatorname{ME} / \operatorname{MEE}(P=.02$, Fig. 5). The use of nonprophylactic antibiotics was also significantly linked to developing worse ME/MEE (nonprophylactic antibiotics $24.0 \%$ vs no nonprophylactic antibiotics $3.9 \%, P<.001$ ). These results are summarized in Table 1.

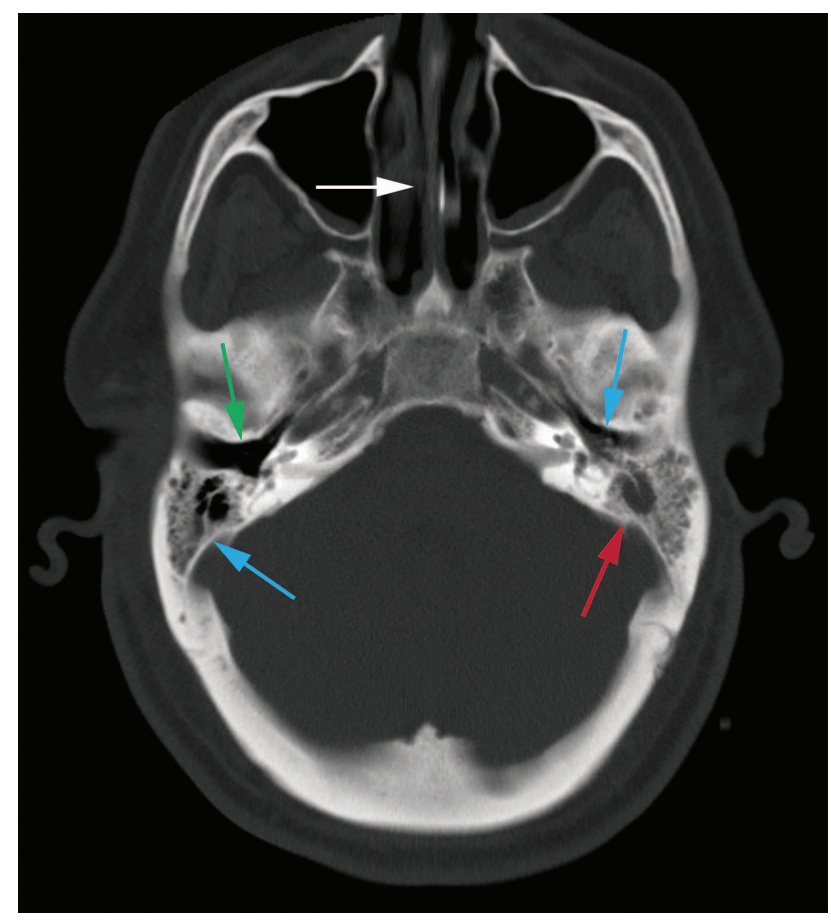

Fig. 1. Computed tomography image demonstrating bilateral mastoid effusions (left complete [red arrow], right partial [blue arrow]) with left middle ear space partial effusion (blue arrow) ipsilateral to a left-sided nasogastric tube (white arrow). The right middle ear space appears normal (green arrow).

There was no statistically significant relationship between developing a worsening effusion and sex (male $10.7 \%$ vs female $9.9 \%, P=.83$ ), immunosuppression $(7.6 \%$ vs no immunosuppression 11.1, $P=.41$ ), or undergoing a surgical procedure (surgery $13.9 \%$ vs no surgery $7.4 \%$, $P=.065$ ). The month of admission was found to have no relationship to the incidence of worsening opacification $(P=.65$, Fig. 6).

Of the subjects who had a nasogastric tube at any point during their stay, $21.4 \%$ (30 of 140) developed ME/MEE compared with $0.6 \%$ (1 of 160) who never had a nasogastric tube $(P<.001)$. The odds ratio $(\mathrm{OR})$ for developing ME/MEE with nasogastric tubes was 43.4 (95\% CI 5.8322.7). At some point during their admission, $22.7 \%$ (30/132) of subjects who had an ETT at any point during their admission developed an ME/MEE. This figure compares with $0.6 \%$ ( 1 of 168) for subjects who never had an ETT $(P<.001)$, representing an OR of 49.1 (95\% CI 6.6-365.7).

Subjects who had both a nasogastric tube and an ETT during their admission developed an ME/MEE 25.4\% (29 of 114) of the time compared with 1.1\% (2 of 186) in those who did not $(P<.001$, OR 31.4, 95\% CI 7.3-134.6). None of the 142 subjects who never had an ETT or nasogastric tube developed worsening effusions. 


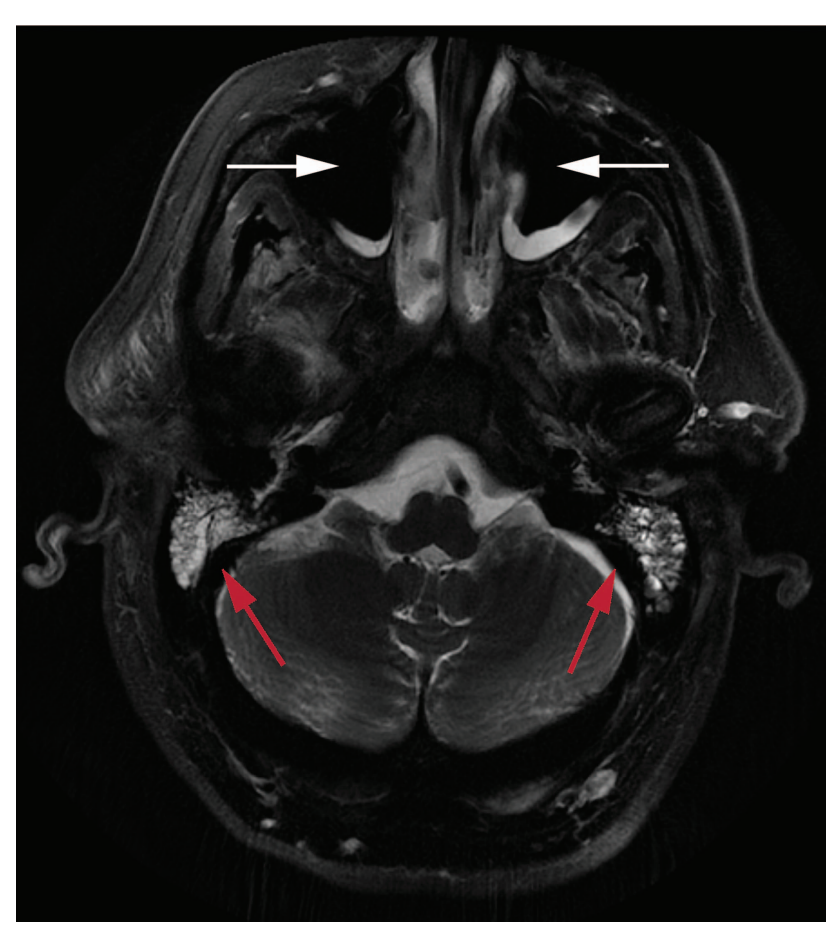

Fig. 2. T2-weighted magnetic resonance imaging demonstrating mastoid effusions (red arrows) and partial maxillary sinus opacification bilaterally (white arrows).

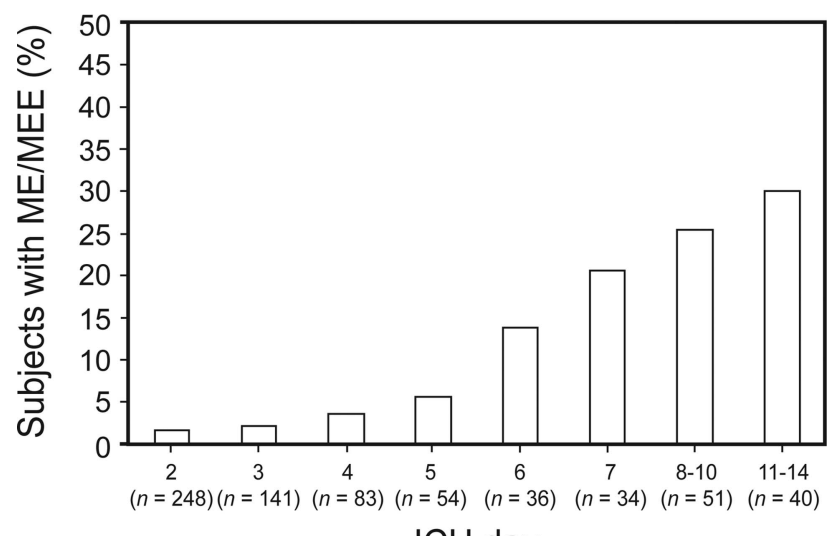

ICU day

Fig. 3. Percentage of subjects developing worsening radiographic mastoid and middle ear effusions by the date of imaging. ME = mastoid effusion, MEE = middle ear effusion.

Given that ETT, nasogastric tubes, and length of stay are probably interrelated, multivariate analysis was performed and revealed that an ETT (OR 10.1, 95\% CI 1.189.0, $P=.037$,$) and a stay >7 \mathrm{~d}(\mathrm{OR} 2.8,95 \% \mathrm{CI}$ $1.0-7.5, P=.043)$ were independently related to the development of ME/MEE. The presence of nasogastric tubes only approached significance (OR 7.5, 95\% CI 0.9-66.4, $P=.069$ ).

At the time of admission, there was no correlation between subjects with radiographic paranasal sinus disease

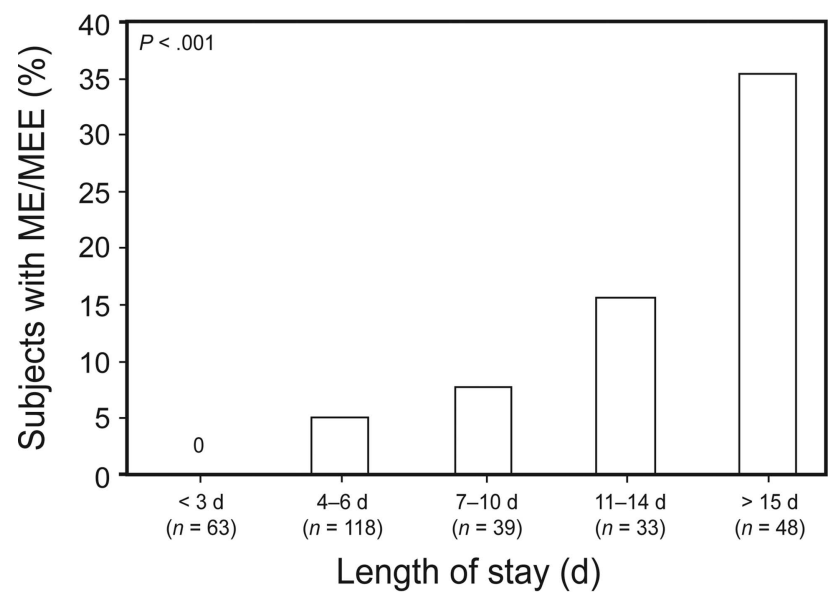

Fig. 4. Percentage of subjects with worsening mastoid or middle ear effusion by length of stay in days. ME = mastoid effusion, $\mathrm{MEE}=$ middle ear effusion.

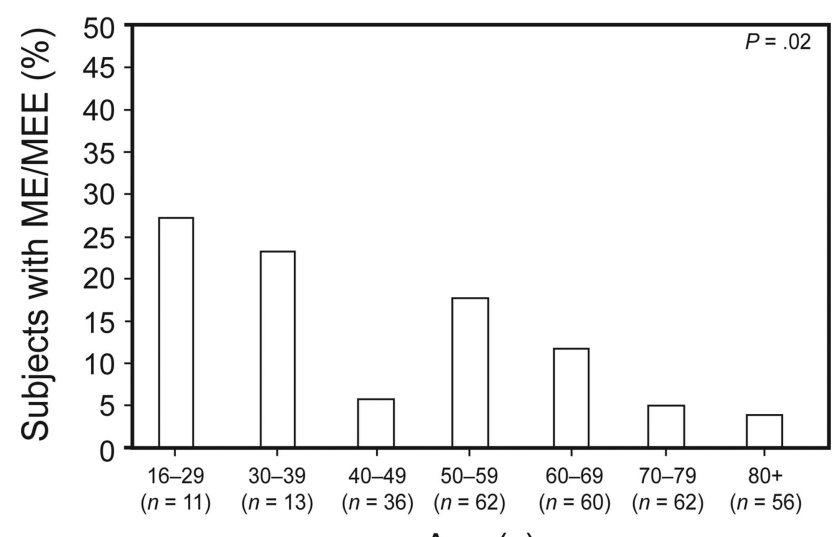

Age $(y)$

Fig. 5. Percentage of subjects with worsening mastoid or middle ear effusion by age. $\mathrm{ME}=$ mastoid effusion, $\mathrm{MEE}=$ middle ear effusion.

and ME/MEE; $3.2 \%$ of subjects with a nonzero LundMcKay score had effusions compared with $4.0 \%$ in subjects with a Lund-McKay score of zero $(P=.73)$. During the ICU admission, however, $90.3 \%$ of subjects ( 28 of 31 ) who developed ME/MEE also developed worsening sinus opacification. Overall, $22.4 \%$ of subjects who developed sinus opacification also developed ME/MEE. This compares with $1.7 \%$ in subjects who did not develop sinus disease $(P<.001)$. The mean highest Lund-McKay score for subjects with ME/MEE was 4.07 versus 2.56 in those without ME/MEE $(P<.001)$.

\section{Discussion}

This study found that 1 in 10 ICU subjects developed radiographically apparent ME and MEEs. Due to the lack of otolaryngologic consultation to evaluate subjects with 
Table 1. Percentage of Subjects With Mastoid and Middle Ear Effusions on Univariate Analysis

\begin{tabular}{lcccc}
\hline \hline \multicolumn{1}{c}{ Characteristics } & Yes $(\%)$ & No $(\%)$ & $P$ & OR (95\% CI) \\
\hline LOS $>7 \mathrm{~d}$ & 23.10 & 3.10 & $<.001$ & $9.3(3.7-23.6)$ \\
Age $>60 \mathrm{y}$ & 6.70 & 15.60 & .01 & $0.39(0.18-0.84)$ \\
Antibiotics & 24.0 & 3.90 & $<.001$ & $7.7(3.3-18.0)$ \\
Male sex & 10.70 & 9.90 & .83 & $1.1(0.52-2.3)$ \\
Immunosuppression & 7.60 & 11.10 & .41 & $0.66(0.24-1.8)$ \\
Surgical procedure & 13.90 & 7.40 & .065 & $2.0(0.95-4.3)$ \\
Endotracheal tube & 22.70 & 0 & $<.001$ & $49.1(6.6-365.7)$ \\
Nasogastric tube & 21.40 & 0.60 & $<.001$ & $43.4(5.8-322.7)$ \\
Sinusitis & 22.40 & 1.70 & $<.001$ & $16.5(4.9-55.9)$ \\
& & & & \\
LOS = length of stay & & & & \\
OR = odds ratio & & & & \\
\hline
\end{tabular}

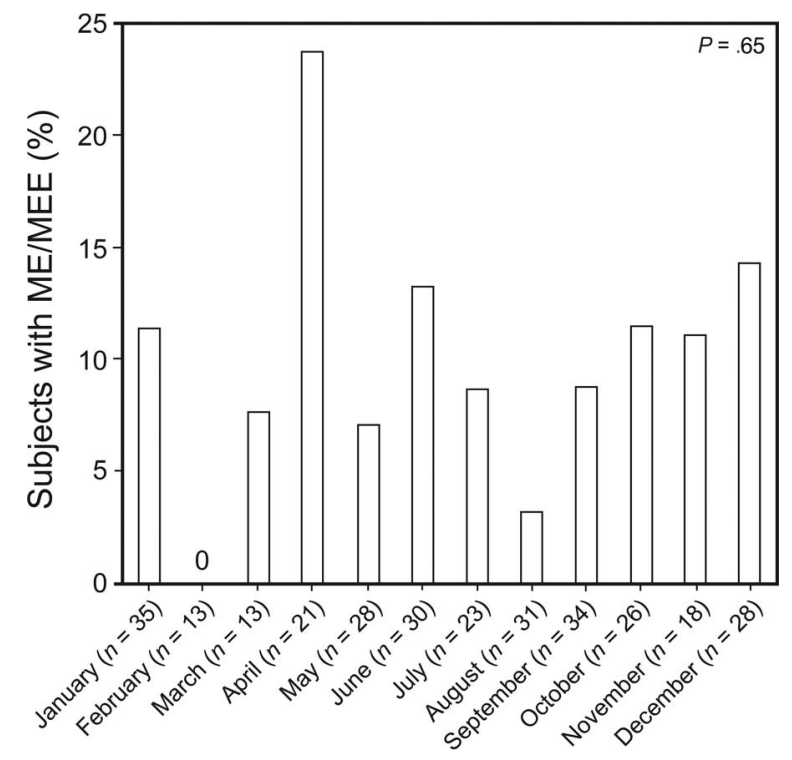

Fig. 6. Percentage of subjects with worsening mastoid or middle ear effusion by month of admission. $M E=$ mastoid effusion, $\mathrm{MEE}=$ middle ear effusion.

these imaging findings or radiographic development of complications related to acute otitis media, we assume that all 31 subjects in this study experienced a benign or sterile ME/MEE. The absence of a relationship to immunosuppression, the nonexistence of seasonal variation (Fig. 6), and positive correlation to antibiotic use further suggest that all cases were benign or sterile ME/MEE.

Nonetheless, the presence of a benign ME/MEE is important to recognize, because of the associated conductive hearing loss in a patient who is less probable to complain of such a symptom because of other competing ailments. We found the rate of ME/MEE to be significantly related to subjects with prolonged lengths of stay and intubation, which are also 2 of the strongest predictors of the development of delirium in the ICU. ${ }^{17,18}$ Hearing loss of any kind contributes to delirium, and minimizing a modifiable cause of sensory impairment, such as ME/MEE, is among the first steps in treating delirium. ${ }^{14}$

When an ME/MEE is incidentally detected on imaging, the treating team should perform otoscopy and question the patient regarding hearing status, if situationally appropriate. Basic measures can be used first, such as increasing vocal projection when addressing the patient and starting intranasal decongestants (oxymetazoline, fluticasone) that can expedite clearance of the effusion through the natural drainage pathway, the Eustachian tube. Although specific institutional patterns will vary, otolaryngologic and/or audiologic consultation is warranted when a middle ear effusion is identified, especially in patients with suspected hearing loss or acute otitis media. A bedside audiogram with tympanometry can diagnose hearing loss and confirm the presence of an effusion. Audiologists at many institutions are also able to provide hearing aids, personal amplification devices, and teach other strategies to assist patients with hearing loss. Otolaryngologists can confirm challenging or uncertain otoscopic findings, and in some cases, may consider offering myringotomy with suctioning and possible pressure equalization tube placement. Myringotomy will often instantly relieve the conductive hearing loss related to middle ear effusion and to a degree can prevent the re-accumulation of fluid, especially if a tube is placed.

The profound effect of ETT and nasogastric tubes on ME/MEE demonstrated here and previously is probably multifactorial. ${ }^{9,10}$ The presence of a foreign body and the associated build-up of retained and thickened secretions probably incite a local inflammatory response of the nasopharyngeal mucosa that narrows and blocks the Eustachian tube. Direct, retrograde flow of secretions and pathogens through the Eustachian tube has also been proposed. Indeed it has been shown that middle ear effusions improve when patients are transitioned to tracheostomy, which is probably related to distancing of the foreign body from the nasopharynx, an improved ability to suction the nose and oral cavity, and lightened level of sedation. ${ }^{19}$

A nasogastric tube may also provide further mucosal edema by allowing gastric contents to reflux along the tube and into the nasopharynx. ${ }^{20}$ Another possible mechanism is direct obstruction of the Eustachian tube orifice by the nasogastric tube, especially if excessive tubing is coiled in the nasopharynx. This explanation is highly unlikely for most cases, however, given the unilaterality of a nasogastric tube and the predominance of bilateral effusions.

By proxy, the presence of nasogastric tubes and especially ETT indicates a depressed level of consciousness (pharmacologically induced or not). This has several implications, most simply an impaired ability to open the Eustachian tubes and clear the middle ear space during 
swallowing or auto-insufflation. Additionally, it has been demonstrated that negative middle ear pressures are associated with even a brief, non-nitrous oxide general anesthetic, including propofol. ${ }^{21}$ It could also be argued that simply lying supine may increase the dependent accumulation of fluid in mastoid or middle ear space. In this study, the low rate of ME/MEE in the nonintubated population $(0.6 \%)$ suggests that the effect of lying supine alone is not sufficient for the development of ME/MEE. Kesser et $\mathrm{al}^{9}$ used a non-ICU population as a control group and similarly found a $>10$-fold decrease in middle ear effusions (5.1\% vs $51.4 \%, P<.01$ ), indicating that lying supine without an ETT does not impart a significant risk of ME/MEE.

Our study found younger age to be correlated with increased risk of ME/MEE, which is in contradistinction to all prior reports. In our population, subjects $<60 \mathrm{y}$ had longer lengths of stay $(9.70$ vs $7.30 \mathrm{~d}, P=.004)$ and higher rates of ETT $(50.8 \%$ vs $39.3 \%, P=.049)$ and nasogastric tubes $(52.5 \%$ vs $42.7 \%, P=.10)$, which perhaps lead to a bias that is unique to our sample of neurologic ICU subjects in comparison with other ICU populations.

The strong positive correlation between ME/MEE and radiographic sinus disease in ICU patients is perhaps not surprising, but has not been specifically reported upon to our knowledge. Sinusitis is a known risk factor for otitis media with effusion in the general adult population, and it appears that this is true of the ICU population as well. ${ }^{22} \mathrm{In}$ this same study cohort, we previously found that $42.6 \%$ of ICU subjects exhibit worsening radiographic sinus opacification, so the strong correlation between radiographic sinusitis and development of ME/MEE puts a significant number of ICU subjects at risk of developing ME/MEE. ${ }^{16}$ Subjects with more severe sinus disease (mean highest Lund-McKay score 4.07 vs $2.56, P<.001$ ) additionally seem to be at a greater risk for effusions in the mastoid and middle ear spaces.

Our study did demonstrate a considerably lower rate of ME/MEE compared with that reported in prior studies $(10.3 \%$ vs $25-80 \%)$ in ICU subjects. ${ }^{6-10}$ This is probably due to several differences, most importantly the population under study and the methodology used to identify the presence of MEE. First, as described above, we considered any subject who remained in the ICU for 2 nights to be critically ill. This perhaps introduced a broader range of illness severity, especially in the neurologic ICU population where a patient may be monitored for a neurologic deficit rather than multisystem organ failure. Other studies focused predominantly on subjects with prolonged intubation, which as we demonstrated is strongly correlated with ME/MEE.

Given the retrospective nature of this study, clinical correlation to subject symptoms, otoscopic exam, and ancillary testing, such as audiography and tympanography, was unfortunately not possible. The differential sensitivity of these diagnostic modalities compared with the review of head imaging with 5-mm slice thickness may further explain the discrepancies in incidence rates reported. With that said, an effusion with clinically important implications on hearing or an infection should appear on head imaging, which as mentioned is frequently performed for other reasons. For this reason, the combination of incidental radiographic review, history, and physical exam should identify relevant otologic effusions and lead to improved care of the recovering ICU patient.

Regardless of the methodology, this and prior studies have demonstrated that a significant portion of ICU patients are at risk for the development of ME/MEE, the true clinical implications of which are unknown. Therefore, there is potentially great value in future prospective research complete with imaging review, physical exam, and audiometric data with correlation to patient factors, such as length of ICU stay and measures of delirium.

\section{Conclusion}

Worsening radiographic middle ear and mastoid effusions were detected in $10.3 \%$ of subjects admitted to the ICU. Factors associated with higher incidence rates included prolonged length of stay, presence of endotracheal tubes, nasogastric tubes, and radiographic sinus disease. In these high-risk groups, the critical care physician and otolaryngologist should take advantage of existing imaging to incidentally diagnose this common finding. Recognition of ME/MEE in the ICU population may promote evaluation for and treatment of any associated hearing loss.

\section{ACKNOWLEDGMENTS}

Special thanks to Li Wang and Dan Winger.

\section{REFERENCES}

1. Bluestone CD, Beery QC, Andrus WS. Mechanics of the Eustachian tube as it influences susceptibility to and persistence of middle ear effusions in children. Ann Otol Rhinol Laryngol. 1974;83:Suppl 11: 27-34.

2. Blomgren K, Robinson S, Lonnqvist T, Saxèn H, Pitkäranta A. Clinical significance of incidental magnetic resonance image abnormalities in mastoid cavity and middle ear in children. Int $\mathrm{J}$ Pediatr Otorhinolaryngol 2003;67(7):757-760.

3. Balci A, Sangun O, Okuyucu S, Karazincir S, Akoglu E, Altintas Y, Egilmez E. The prevalence and significance of incidental middle ear and mastoid cavity abnormalities on MRI in a pediatric population. Int J Pediatr Otorhinolaryngol 2008;72(12):1849-1854.

4. von Kalle T, Fabig-Moritz C, Heumann H, Winkler P. Incidental findings in paranasal sinuses and mastoid cells: a cross-sectional magnetic resonance imaging (MRI) study in a pediatric radiology department. Rofo 2012;184(7):629-634.

5. Lee DH, Jun BC, Park JO, Yeo SW. Magnetic resonance imaging of the mastoid cavity and middle ear: prevalence and clinical significance of incidental abnormal findings in a nonotolaryngologic adult and pediatric population. J Otolaryngol 2006;35(1):13-18. 


\section{RADIOGRAPHIC ME AND MEE IN ICU SUBJECTS}

6. Lin CC, Lin CD, Cheng YK, Tsai MH, Chang CS. Middle ear effusion in intensive care unit patients with prolonged endotracheal intubation. Am J Otolaryngol 2006;27(2):109-111.

7. Christensen L, Schaffer S, Ross SE. Otitis media in adult trauma patients: incidence and clinical significance. J Trauma 1991;31(11): 1543-1545.

8. Cavaliere F, Masieri S, Liberini L, Proietti R, Magalini SI. Tympanometry for middle-ear effusion in unconscious ICU patients. Eur J Anaesthesiol 1992;9(1):71-75.

9. Kesser BW, Woodard CR, Stowell NG, Becker SS. Middle ear effusion in adult ICU patients: a cohort study. Ear Nose Throat J 2013;92(8):340-346.

10. González Pena M, Figuerola Massana E, Hernández Gutierrez P, Rello Condomines J. Middle ear effusion in mechanically ventilated patients: effects of the nasogastric tube. Respir Care 2013;58(2):273278.

11. Salibi PN, Agarwal V, Panczykowski DM, Puccio AM, Sheetz MA, Okonkwo DO. Lifetime attributable risk of cancer from CT among patients surviving severe traumatic brain injury. AJR Am J Roentgenol 2014;202(2):397-400.

12. Persico M, Barker GA, Mitchell DP. Purulent otitis media-a "silent" source of sepsis in the pediatric intensive care unit. Otolaryngol Head Neck Surg 1985;93(3):330-334.

13. Derkay CS, Bluestone CD, Thompson AE, Kardatske D. Otitis media in the pediatric intensive care unit: a prospective study. Otolaryngol Head Neck Surg 1989;100(4):292-299.
14. Fong TG, Tulebaev SR, Inouye SK. Delirium in elderly adults: diagnosis, prevention and treatment. Nat Rev Neurol 2009;5(4):210220.

15. Lund VJ, Mackay IS. Staging in rhinosinusitus. Rhinology 1993; 31(4):183-184.

16. Huyett P, Lee S, Ferguson BJ, Wang EW. Sinus opacification in the intensive care unit patient. Laryngoscope 2016;26(11):2433-2438.

17. Van Rompaey B, Elseviers MM, Schuurmans MJ, Shortridge-Baggett LM, Truijen S, Bossaert L. Risk factors for delirium in intensive care patients: a prospective cohort study. Crit Care 2009;13(3):R77.

18. Zaal IJ, Devlin JW, Peelen LM, Slooter AJ. A systematic review of risk factors for delirium in the ICU. Crit Care Med 2015;43(1): 40-47.

19. Chung HK, Lin CC, Wang CY, Lin CD, Tsai MH, Chang CS. Improvement in otitis media with effusion in patients undergoing tracheostomy after prolonged endotracheal intubation. J Otolaryngol Head Neck Surg 2009;38(5):532-536.

20. Dotson RG, Robinson RG, Pingleton SK. Gastroesophageal reflux with nasogastric tubes. Effect of nasogastric tube size. Am J Respir Crit Care Med 1994;149(6):1659-1662.

21. Kanai R, Kaneko K. Negative middle ear pressure and otitis media with effusion after surgery under general anesthesia. Acta Otolaryngol 2012;132(10):1049-1053.

22. Shimotakahara SG, Ruby RR, Lampe HB. Otitis media with effusion in the adult. J Otolaryngol 1989;18(3):85-89. 